\title{
Magnetic properties of transition metal dichalcogenides-Fe/Ir(111) interfaces from first principles
}

\author{
Vasile Caciuc, ${ }^{*}$ Nicolae Atodiresei, ${ }^{\dagger}$ and Stefan Blügel \\ Peter Grünberg Institut (PGI-1) and Institute for Advanced Simulation (IAS-1), Forschungszentrum Jülich and JARA, \\ D-52425 Jülich, Germany
}

(Received 16 May 2018; published 6 August 2018)

\begin{abstract}
In our density functional theory study we systematically explored how the interaction between magnetic and nonmagnetic transition metal dichalcogenide (TMD) monolayers and a magnetic Fe/Ir(111) electrode reciprocally modifies their electronic and magnetic properties. From an electronic point of view, all investigated semiconductor TMDs become metallic upon adsorption on the Fe/Ir(111) substrate related to a rather strong TMD-Fe/Ir(111) hybridization. On the other hand, from a magnetic point of view, the TMD-surface hybridization leads to a spin-imbalanced electronic structure of such hybrid systems with system-specific features. Additionally, the calculated exchange coupling constants for the Fe surface atoms evidenced a stronger magnetic interaction upon TMD adsorption as compared to the case of the clean surface. This observation indicates the presence of a magnetic hardening of the exchange coupling constants similarly to the case of molecular systems on magnetic surfaces, which emphasizes the generality of this adsorbate-induced effect on magnetic substrates.
\end{abstract}

DOI: 10.1103/PhysRevMaterials.2.084001

\section{INTRODUCTION}

Recently, the two-dimensional (2D) transition metal dichalcogenides (TMDs) emerged as a promising technological alternative [1-4] to the graphene-based electronic and spintronic devices [5,6]. For instance, a single-layer $\mathrm{MoS}_{2}$ was successfully employed as the active channel in a field effect transistor (FET) [7], a direct gap semiconductor $\mathrm{WS}_{2}$ monolayer in optoelectronic devices [8,9], or bilayer $\mathrm{MoS}_{2}$ transistors in integrated circuits [10].

In bulk the layered TMDs described by the chemical formula $M X_{2}$ ( $M$ is a transition metal from the group IV, $\mathrm{V}$, or VI and $X$ is a chalcogen one) exhibit a hexagonal $X-M-X$ layer structure with covalent $X-M$ bonds bound by the van der Waals interlayer interactions [1]. Importantly, for semiconducting TMDs the transition from the bulk to monolayer semiconductor TMDs is associated with a change from indirect to direct band gap with a significant impact on their optical properties [11]. Moreover, the electronic structure of single TMD layers can be subsequently tuned by a chemical functionalization process [12]. In particular, TMD monolayers that lack the spatial inversion symmetry open the fascinating prospect of coupling the spin and valley degrees of freedom via spin-orbit coupling (SOC) [13] leading to valleytronics devices for optoelectronic applications $[14,15]$. Furthermore, it was experimentally shown that the valley pseudospin can be tuned via a magnetic field [16] and the strength of SOC can also be tailored by alloying a $\mathrm{MoSe}_{2}$ monolayer with tungsten (W) to design optoelectronic devices with an efficient valley polarization [17]. Besides this, ab initio simulations were employed to model large-gap quantum spin Hall materials based on TMDs [18].

\footnotetext{
*v.caciuc@fz-juelich.de

†n.atodiresei@fz-juelich.de
}

From a technological point of view, the TMD-metal surface interface significantly controls the performance of a TMDbased electronic device through the contact resistance $[4,19-$ 21]. In this regard, for a $\mathrm{MoS}_{2}$ monolayer on the $\operatorname{Ir}(111)$, $\mathrm{Pd}(111)$, and $\mathrm{Ru}(0001)$ surfaces a Schottky-type barrier was unveiled from first principles [22] and similar results were obtained for single-layer TMDs such as $\mathrm{MoS}_{2}, \mathrm{VS}_{2}, \mathrm{MoSe}_{2}$, and $\mathrm{WSe}_{2}$ on other metal surfaces [23-28].

More importantly, to explore the perspective of using TMD in spintronic devices [29] it is essential to analyze how the magnetic properties of single TMD layers can be specifically engineered due to their interaction with a metal substrate of choice. In this respect, it was experimentally shown that the valley polarization in $\mathrm{WSe}_{2}$ can be induced via spin injection from a permalloy ferromagnetic electrode in a light-emitting diode [30]. In addition, the spin-valve effect was evidenced in experiments performed for a monolayer $\mathrm{MoS}_{2}$ acting as a nonmagnetic spacer in $\mathrm{NiFe} / \mathrm{MoS}_{2} / \mathrm{NiFe}$ heterostructures [31] and a similar spin-dependent transport behavior was revealed for $\mathrm{Fe}_{3} \mathrm{O}_{4} / \mathrm{MoS}_{2} / \mathrm{Fe}_{3} \mathrm{O}_{4}$ magnetic tunnel junctions [32]. From a theoretical point of view, several studies focused especially on the electronic properties of the intrinsic magnetic $\mathrm{VS}_{2}$ and $\mathrm{VSe}_{2}$ monolayers [33,34], in TMD nanoribbons [35,36], in hydrogenated [37] and fluorinated [38] single TMD layers, and in TMD monolayers doped with transition metal atoms [39-41] or strain-induced ones [42-44]. On the other hand, less attention was focused on the properties of TMDs on magnetic substrates [45-50] that can serve, for instance, as electrodes in spintronic devices [51].

Therefore in this first-principles study we systematically investigate the electronic and magnetic properties of hybrid systems formed by nonmagnetic $(M=\mathrm{Mo}, \mathrm{W}$ and $X=\mathrm{S}, \mathrm{Se})$ and magnetic $(M=\mathrm{V}$ and $X=\mathrm{S}, \mathrm{Se}) \mathrm{TMD}$ monolayers adsorbed on the Fe/Ir(111) surface. Regarding the Fe/Ir(111) substrate, it is important to note that although the $\mathrm{Fe}$ monolayer on $\operatorname{Ir}(111)$ exhibits a complex magnetic structure [52], in a recent 
combined experimental and theoretical work we demonstrated that the chemisorption of finite (molecular) and extended (graphene nanoislands) $\pi$ systems on this substrate leads to a local ferromagnetic order of the Fe surface atoms [53]. On this basis we focused on (i) how the electronic and magnetic properties of the TMD layers can be engineered in the presence of a $3 d$ magnetic layer and conversely (ii) how the magnetic properties of the Fe layer are modulated by its interaction with the TMD monolayers.

In particular, our $a b$ initio study revealed that the semiconductor TMDs are chemisorbed on the magnetic substrate and exhibit a metallic character due to a significant TMD$\mathrm{Fe} / \mathrm{Ir}(111)$ hybridization. This TMD-substrate hybridization is spin-dependent and determines a system-specific spinimbalanced electronic structure of the corresponding hybrid systems. In this respect, a strong hybridization between the $d$ states of the surface $\mathrm{Fe}$ and $\mathrm{V}$ transition metal atoms via the $p$ states of the interfacial $\mathrm{S}(\mathrm{Se})$ atomic layer leads a larger spin-polarized density of states at the Fermi energy for the Vbased hybrid systems as compared to the nonmagnetic TMDs. Particularly, this feature may play an important role in the spin transport in these systems. Furthermore, similarly to the way finite and extended $\pi$ systems modify the magnetic properties of metal surfaces [53-57], the calculated exchange coupling constants $J$ describing the strength of the magnetic interactions between the surface Fe atoms also exhibit a magnetic hardening due to the adsorbate-magnetic substrate hybridization effects.

\section{COMPUTATIONAL SETUP}

Our first-principles calculations were carried out using density functional theory (DFT) [58,59] with the generalized gradient approximation [60] for the exchange-correlation energy functional. The interaction between the valence electrons and the atomic cores is described by pseudopotentials generated with the help of the projector augmented wave method (PAW) [61] as implemented in the VASP code [62-64]. As transition metal dichalcogenide (TMD) single layers, in our $a b$ initio study the $\mathrm{V}, \mathrm{Mo}$, and $\mathrm{W}$ disulphides and diselenides were considered (i.e., $\mathrm{VS}_{2}, \mathrm{MoS}_{2}, \mathrm{WS}_{2}, \mathrm{VSe}_{2}, \mathrm{MoSe}_{2}$, and $\mathrm{WSe}_{2}$, respectively) with hexagonal symmetry. To minimize the TMD-surface lattice mismatch [22], the corresponding six TMD/Fe/Ir(111) systems were modeled by a $\sqrt{3} \times \sqrt{3}$ TMD surface unit cell on the $2 \times 2 \operatorname{Ir}(111)$ surface consisting of five atomic layers. Additionally, the vacuum region separating the topmost TMD layer and the next slab was larger than 18 $\AA$. Furthermore, the atomic degrees of freedom of the TMD, $\mathrm{Fe}$, and first Ir surface layer were relaxed until the calculated Hellmann-Feynman forces were smaller than $5 \mathrm{meV} / \AA \mathrm{ob}$ tained for a $k$ mesh of $14 \times 14 \times 1$ and plane waves basis set determined by a cutoff energy of $500 \mathrm{eV}$.

\section{RESULTS}

As depicted in Fig. 1(a), in the ground state the $\mathrm{VS}_{2}$ and $\mathrm{VSe}_{2}$ monolayers adsorb in a $\mathrm{S}_{\text {top }}-\mathrm{S}_{\text {hollow }}\left(\mathrm{Se}_{\text {top }}-\mathrm{Se}_{\text {hollow }}\right)$ geometry characterized by the $\mathrm{S}(\mathrm{Se})$ atoms of the lower TMD atomic layer above Fe and on the top and hcp surface Ir atoms. On the other hand, for the Mo and $\mathrm{W}$ disulphide and diselenide TMDs the $\mathrm{S}$ and $\mathrm{Se}$ atoms are in an atop adsorption position

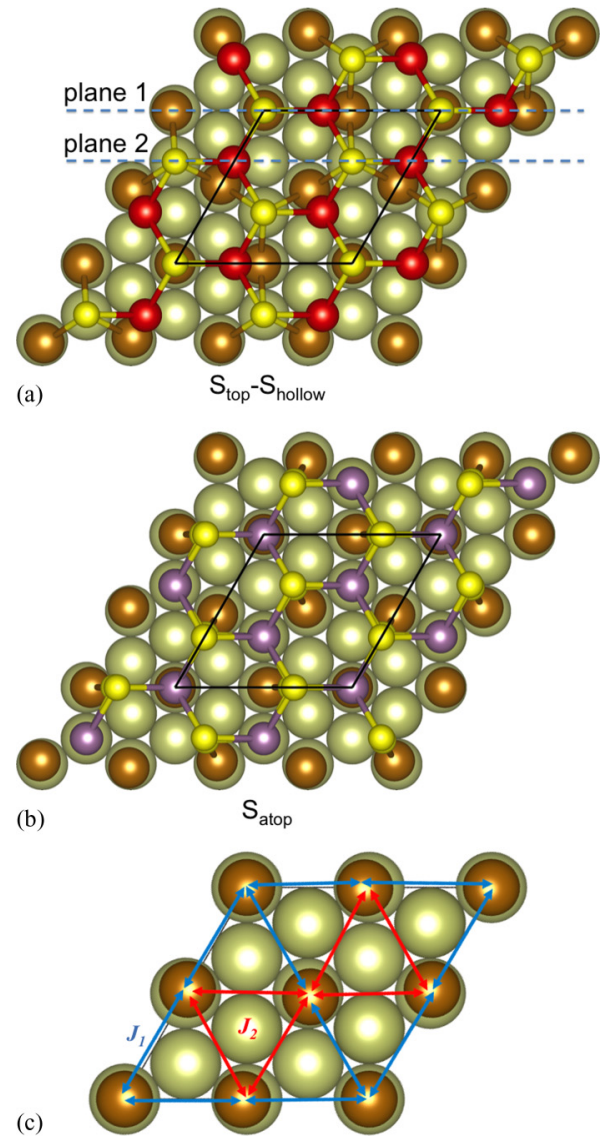

FIG. 1. The ground-state adsorption geometry for the (a) $\mathrm{VS}_{2}$ $\left(\mathrm{VSe}_{2}\right)$ and (b) Mo and $\mathrm{W}$ disulphide and diselenide single layers on $\mathrm{Fe} / \mathrm{Ir}(111)$. In the case of the $\mathrm{VS}_{2}-$ and $\mathrm{VSe}_{2} / \mathrm{Fe} / \mathrm{Ir}(111)$ systems the chalcogen atoms prefer top ( $\mathrm{Fe}_{\text {surf }}^{\mathrm{S} / \mathrm{Se}}$ and $\mathrm{Ir}$ ) and hollow (Ir) adsorption sites while they are in an atop adsorption configuration with respect to the surface $\mathrm{Fe}$ atoms (denoted also as $\mathrm{Fe}_{\text {surf }}^{\mathrm{S} / \mathrm{Se}}$ ) for the Mo- and W-based systems. The plane 1 (containing $\mathrm{S}, \mathrm{V}$, and $\mathrm{Fe}$ atoms) and 2 (holding $\mathrm{S}$ and $\mathrm{V}$ atoms) in (a) are the planes used to plot the spin polarization in Fig. 3. (c) The exchange coupling constants $J_{1}$ and $J_{2}$ that describe within the Heisenberg Hamiltonian model the magnetic interactions between the surface Fe atoms (see text).

with respect to the Fe magnetic layer [see Fig. 1(b)]. Overall, in both cases one can differentiate between the Fe atoms underneath the $\mathrm{S}$ and $\mathrm{Se}$ ones in a top or atop configuration (denoted in the following as $\mathrm{Fe}_{\text {surf }}^{\mathrm{S} / \mathrm{Se}}$ ) and the remaining ones $\mathrm{Fe}_{\text {surf }}$ (the corresponding Fe-S/Se bond lengths are presented in Table I). Regarding the strength of the TMD-substrate interaction, the calculated adsorption energies reported in Table I clearly indicate that the TMDs containing $\mathrm{S}$ atoms are more strongly chemisorbed on $\mathrm{Fe} / \mathrm{Ir}(111)$ than those incorporating $\mathrm{Se}$ atoms. In addition, we also note that for both $\mathrm{S}$ - and $\mathrm{Se}$ based TMDs the largest adsorption energy is obtained for the hybrid TMD-surface systems containing V atoms. As a direct consequence, the TMD-surface hybridization is expected to lead to TMD-specific features in the electronic structure of the hybrid systems investigated in our first-principles study.

Indeed, as presented in Fig. 2 for the $d$ states of the TM atoms of the TMD monolayers, close to the Fermi energy $E_{F}$ the calculated spin-polarized atom-projected density of states 
TABLE I. The calculated adsorption energy $E_{\text {ads }}$ defined as $E_{\text {ads }}=E_{\text {sys }}-\left(E_{\text {surf }}+E_{\mathrm{TMD}}\right)$, where $E_{\text {sys }}$ is the total energy of the TMD-surface hybrid system, $E_{\text {surf }}$ represents the total energy of the $\mathrm{Fe} / \mathrm{Ir}(111)$ surface, and $E_{\mathrm{TMD}}$ corresponds to the total energy of the freestanding $\sqrt{3} \times \sqrt{3}$ TMD monolayer. The TM-chalcogen distances between the surface $\mathrm{Fe}_{\text {surf }}^{\mathrm{S} / \mathrm{Se}}$ atoms underneath the $\mathrm{S}$ (Se) and the remaining Fe $E_{\text {surf }}$ ones are also presented. Additionally, the magnetic moments of the interfacial chalcogen ( $\mathrm{S}$ or $\mathrm{Se}$ ) and TM (V, Mo, W, and Fe) atoms as well as the calculated magnetic exchange coupling constants $J$ for the surface Fe atoms [see Fig. 1(c)] are reported. More precisely, the exchange coupling constants $J_{1}$ and $J_{2}$ describe the magnetic exchange interactions of $\mathrm{Fe}_{\text {surf }}^{\mathrm{S} / \mathrm{Se}}-\mathrm{Fe}_{\text {surf }}$ and $\mathrm{Fe}_{\text {surf }}^{\mathrm{S}}-\mathrm{Fe}_{\text {surf }}^{\mathrm{S} / \mathrm{Se}}$ (or $\mathrm{Fe}_{\text {surf }}$ coordinated by $\mathrm{S} / \mathrm{Se}$ in top-hollow sites) atomic pairs, respectively. As a reference, the exchange coupling constant of the clean Fe/ $\operatorname{Ir}(111)$ surface $J_{\mathrm{cs}}$ is $18.0 \mathrm{meV}$ [53]. Then, overall, a hardening of the calculated $J$ values is obtained for all investigated hybrid TMD/Fe/Ir(111) systems.

\begin{tabular}{|c|c|c|c|c|c|c|c|c|c|}
\hline \multirow[b]{2}{*}{ System } & \multirow{2}{*}{$\begin{array}{l}E_{\text {ads }} \\
(\mathrm{eV})\end{array}$} & \multicolumn{2}{|c|}{ Fe-S/Se bond length $(\AA)$} & \multicolumn{4}{|c|}{$\begin{array}{l}\text { Magnetic moment } \\
\qquad\left(\mu_{B}\right)\end{array}$} & \multicolumn{2}{|c|}{$\begin{array}{c}\text { Exchange coupling } \\
\text { constant } J(\mathrm{meV})\end{array}$} \\
\hline & & $\mathrm{Fe}_{\text {surf }}^{\mathrm{S} / \mathrm{Se}}-\mathrm{S} / \mathrm{Se}$ & $\mathrm{Fe}_{\text {surf }}-\mathrm{S} / \mathrm{Se}$ & $\mathrm{TM}$ & $\mathrm{S} / \mathrm{Se}$ & $\mathrm{Fe}_{\text {surf }}^{\mathrm{S} / \mathrm{Se}}$ & $\mathrm{Fe}_{\text {surf }}$ & $J_{1}$ & $J_{2}$ \\
\hline $\mathrm{VS}_{2} / \mathrm{Fe} / \operatorname{Ir}(111)$ & -3.46 & 2.18 & 2.43 & 0.33 & 0.03 & 2.38 & 2.66 & 19.74 & 30.35 \\
\hline $\mathrm{MoS}_{2} / \mathrm{Fe} / \mathrm{Ir}(111)$ & -1.75 & 2.27 & 2.82 & -0.10 & 0.02 & 2.51 & 2.86 & 29.41 & 23.73 \\
\hline $\mathrm{WS}_{2} / \mathrm{Fe} / \mathrm{Ir}(111)$ & -1.35 & 2.30 & 2.88 & -0.07 & 0.02 & 2.52 & 2.83 & 28.00 & 27.22 \\
\hline $\mathrm{VSe}_{2} / \mathrm{Fe} / \mathrm{Ir}(111)$ & -1.94 & 2.34 & 2.59 & 0.21 & 0.03 & 2.50 & 2.71 & 22.35 & 33.94 \\
\hline $\mathrm{MoSe}_{2} / \mathrm{Fe} / \operatorname{Ir}(111)$ & -0.87 & 2.46 & 2.99 & -0.02 & 0.02 & 2.60 & 2.83 & 23.67 & 30.12 \\
\hline $\mathrm{WSe}_{2} / \mathrm{Fe} / \mathrm{Ir}(111)$ & -0.57 & 2.49 & 3.04 & -0.02 & 0.01 & 2.61 & 2.81 & 21.82 & 32.73 \\
\hline
\end{tabular}

(SP-PDOS) indicates a significantly larger density of electronic states in the case of $\mathrm{VS}_{2}$ and $\mathrm{VSe}_{2}$ on $\mathrm{Fe} / \mathrm{Ir}(111)$ as compared with the nonmagnetic TMDs for both spin channels. On the other hand, close to the Fermi energy $E_{F}$ the SP-PDOS for the $\mathrm{WS}_{2}\left(\mathrm{WSe}_{2}\right)$ exhibits similar features to those for $\mathrm{MoS}_{2}$ $\left(\mathrm{MoSe}_{2}\right)$ (see also the Supplementary Material (SM) [65]). Besides this, one can also observe spin-imbalanced features in the calculated SP-PDOS for all investigated TMDs that suggest a system-dependent spin polarization at the hybrid interface. Finally, it is also important to mention that all semiconductor
TMDs considered in this study exhibit a finite PDOS at $E_{F}$ implying that they become metallic upon adsorption on the $\mathrm{Fe} / \mathrm{Ir}(111)$ surface (see also SM [65]). This observation is also clearly supported by the comparison with the SP-PDOS obtained for TM $d$ states of the corresponding freestanding $\sqrt{3} \times \sqrt{3}$ TMD monolayers (see Fig. 2).

Additionally, close to $E_{F}$ the SP-PDOS evaluated for the transition metal atoms of the TMD single layers investigated in our theoretical study (see Fig. 2) and that for the Fe layer (see Fig. 1 in the SM [65]) is significantly larger in the case

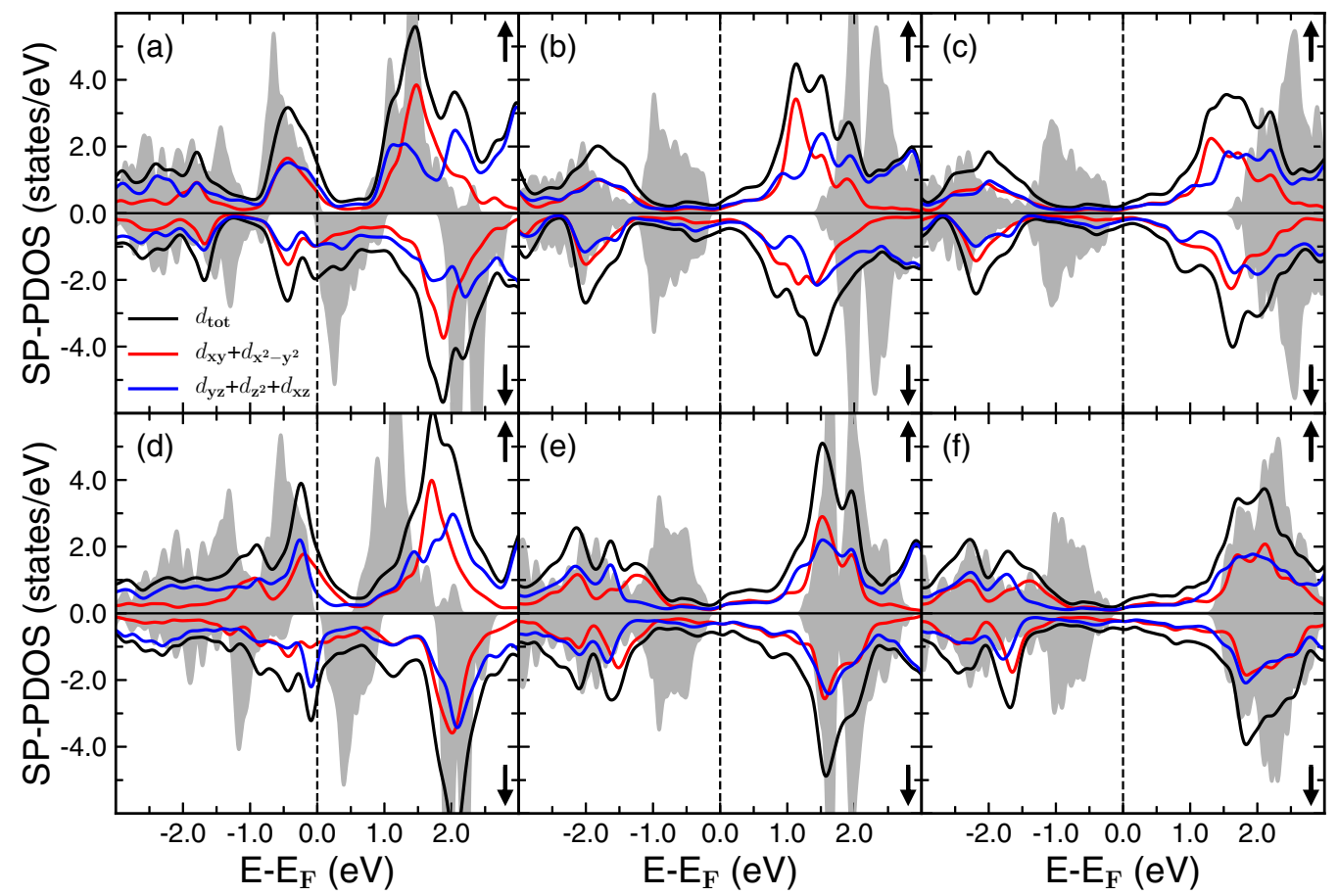

FIG. 2. The spin-polarized atom-projected density of states (SP-PDOS) for the total, in-plane $\left(d_{x y}+d_{x^{2}-y^{2}}\right)$, and out-of-plane $\left(d_{y z}+d_{z^{2}}+\right.$ $d_{x z}$ ) $d$ states of the TM layer in the case of (a) $\mathrm{VS}_{2}$, (b) $\mathrm{MoS}_{2}$, (c) $\mathrm{WS}_{2}$, (d) $\mathrm{VSe}_{2}$, (e) $\mathrm{MoSe}_{2}$, and (f) WSe 2 on Fe/Ir(111). Note that in gray are depicted the corresponding TM total $d$ states of the freestanding $\sqrt{3} \times \sqrt{3}$ TMD monolayers. It is also important to mention that a strong spin-dependent hybridization between $\mathrm{V} d$ states with the substrate ones leads to a spin-unbalanced electronic structure near Fermi energy while this feature is less pronounced for Mo and W transition metal atoms. 


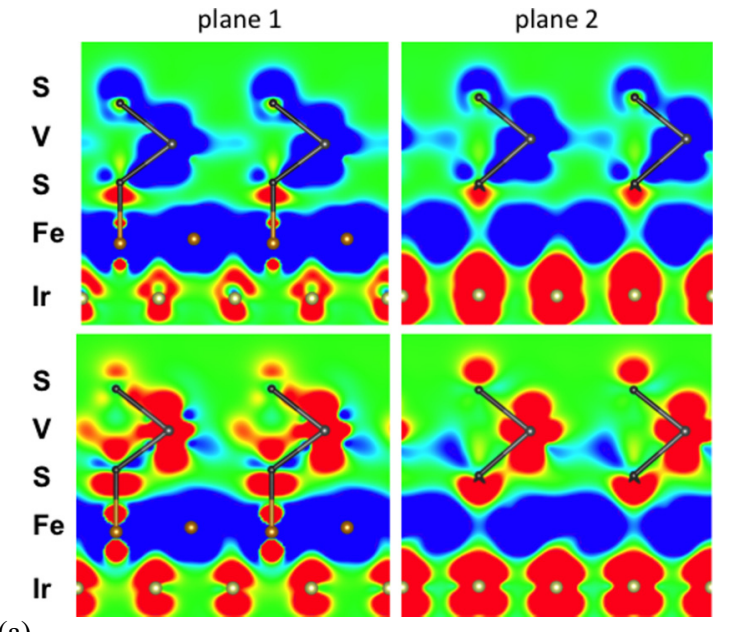

(a) (b)

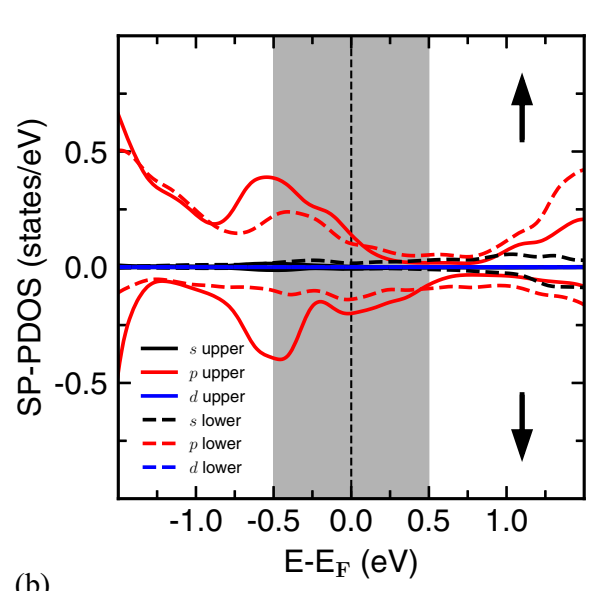

)

FIG. 3. (a) The spin polarization distribution in space for $\mathrm{VS}_{2}$ on $\mathrm{Fe} / \mathrm{Ir}(111)$ plotted in the planes indicated in Fig. 1. The images in the lower panel were obtained for the occupied electronic states in the energy interval $\left[E_{F}-0.5 \mathrm{eV}, E_{F}\right]$ while the upper ones correspond to the unoccupied electronic states in the energy interval $\left[E_{F}, E_{F}+0.5 \mathrm{eV}\right]$. Overall, note that a negative spin density is delocalized over the Fe layer while a positive spin polarization is present at the lower interface $S$ and first Ir layer atoms. However, the upper panel clearly indicates a negative spin polarization at the $\mathrm{V}$ and upper $\mathrm{S}$ layer atoms (for the unoccupied electronic states) and a predominantly positive one in the case of the occupied electronic states (lower panel). The negative spin density is shown in blue and the positive one in red and yellow. (b) These features of the spin polarization for $\mathrm{VS}_{2}$ on $\mathrm{Fe} / \mathrm{Ir}(111)$ are directly reflected by the SP-PDOS calculated for the upper and lower S layer (for more details see Fig. 2 in the Supplemental Material [65]). More specifically, the analysis of the SP-PDOS for the upper S layer shows that the difference between the spin-up and spin-down electronic states is positive for the interval $\left[E_{F}-0.5 \mathrm{eV}, E_{F}\right]$ and negative for the $\left[E_{F}, E_{F}+0.5 \mathrm{eV}\right]$ one.

of the magnetic $\mathrm{VS}_{2}$ and $\mathrm{VSe}_{2}$ monolayers as compared to the nonmagnetic TMDs that may play an important role in the spin transport in these systems. This observation is further supported by a spin-unbalanced electronic structure of the $S$ (Se) atomic layers near Fermi energy (see Fig. 2 in the SM [65]).

Moreover, for the $\mathrm{VS}_{2} / \mathrm{Fe} / \mathrm{Ir}(111)$ system the spatial distribution of the spin polarization corresponding to its spin-split electronic structure in an energy interval of $\left[E_{F}-0.5 \mathrm{eV}, E_{F}\right]$ and $\left[E_{F}, E_{F}+0.5 \mathrm{eV}\right]$ is depicted in the lower and upper panels of Fig. 3(a), respectively. Interestingly, as a general feature, it shows a negative spin polarization delocalized over the Fe layer (in the minority spin channel) while a positive spin polarization (in the majority spin channel) is localized at the lower interface $\mathrm{S}$ and first Ir layer atoms. This observation suggests that two different channels are available for the spin transport in the Fe layer: (1) a localized one at the Fe atoms that form a chemical bond with $\mathrm{S}$ atoms and has an out-of-plane $3 d_{z^{2}}$-like character and (2) a delocalized one over the whole $\mathrm{Fe}$ atomic layer originating from the in-plane $\left(d_{x y}+d_{x^{2}-y^{2}}\right)$ and out-of-plane $\left(d_{y z}+d_{x z}\right) 3 d$-like atomic orbitals. In particular, these features can be of technological interest since the analysis of the plane-averaged electrostatic potential at the $\mathrm{VS}_{2}$ and $\mathrm{VSe}_{2} / \mathrm{Fe} / \mathrm{Ir}(111)$ interfaces suggests the formation of an Ohmic contact while for the remaining TMD-surface hybrid systems an interface tunneling barrier is present (see Fig. 3 in the Supplemental Material [65]).

On the other hand, the upper panel of Fig. 3(a) clearly indicates a negative spin polarization at the $\mathrm{V}$ and upper $\mathrm{S}$ layer atoms (for the unoccupied electronic states) and a predominantly positive one in the case of the occupied electronic states (lower panel). This observation is further supported by the analysis of the SP-PDOS calculated for the upper S layer [see Fig. 3(b)] that reveals a positive difference for the occupied spin-up and spin-down electronic states in the energy interval $\left[E_{F}-0.5 \mathrm{eV}, E_{F}\right]$ and a negative one for the unoccupied states in $\left[E_{F}, E_{F}+0.5 \mathrm{eV}\right]$. Generally, this outcome of our $a b$ initio study implies that in a spin-polarized scanning tunneling microscopy (SP-STM) experiment an opposite spin contrast should be obtained in the recorded topography images when this system is imaged for such an energy interval.

Overall, this behavior of the magnetic TMDs on Fe/Ir(111) is related to a strong spin-dependent hybridization between the TMD and Fe $d$ states such that for the V-based systems close to the Fermi energy a significant part of the unoccupied minority $d$ states of the freestanding TMD layers are shifted below $E_{F}$. It is also worth mentioning that a similar spin-dependent hybridization mechanism was put forward also for the spin injection at hybrid organic-metal interfaces $[66,67]$.

As a direct consequence of the hybridization between the TMD and the substrate electronic states, in the case of $\mathrm{VS}_{2}$ each $\mathrm{V}$ atom has a magnetic moment of $\sim 0.33 \mu_{B}$ while for $\mathrm{VSe}_{2}$ this value is further reduced to $\sim 0.21 \mu_{B}$ being significantly smaller than the corresponding values for the freestanding monolayers $\left(1.0 \mu_{B}\right.$ for $\mathrm{VS}_{2}$ and $1.08 \mu_{B}$ for $\mathrm{VSe}_{2}$, respectively) (see Table I). Additionally, for both Vbased hybrid systems the two chalcogen atoms in a hollow adsorption site at the TMD-substrate interface exhibit a small magnetic moment of $\sim 0.03 \mu_{B}$ while those at the top of the surface $\mathrm{Fe}$ atoms or at the vacuum interface have a negligible one. Similar features are present for the Mo- and W-based TMD monolayers with a smaller average induced magnetic moment for the chalcogen atoms in disulphide TMDs $\left(\sim 0.02 \mu_{B}\right)$ and even smaller for the diselenides.

More importantly, the spin-dependent interaction of the nonmagnetic TMD with the magnetic $\mathrm{Fe} / \operatorname{Ir}(111)$ substrate 
induces a small magnetic moment in the Mo and $\mathrm{W}$ transition metal atoms that are ferromagnetically coupled. In particular, the magnitude of the induced magnetic moment of $0.10 \mu_{B}$ in the case of the $\mathrm{MoS}_{2}$ is similar to that obtained for this TMD on $\operatorname{Gd}(0001)$ [45]. Interestingly, note also that in the V-based hybrid systems the TM atoms couple ferromagnetically to the Fe magnetic layer while an antiferromagnetic order is observed for the induced magnetic moments of the Mo and W atoms as well as of the chalcogen ones due to $\mathrm{Fe}_{\text {surf }}^{\mathrm{S} / \mathrm{Se}}$-interfacial $\mathrm{S}$ (Se) hybridization effects.

On the other hand, the average magnetic moment of the surface $\mathrm{Fe}_{\text {surf }}$ atoms is $\sim 2.78 \mu_{B}$, sizeably larger than in the bulk Fe $\left(\sim 2.2 \mu_{B}\right)$. Nevertheless, the $\mathrm{Fe}_{\text {surf }}^{\mathrm{S} / \mathrm{Se}}$ atoms that locally form a chemical bond with the $\mathrm{S}$ or Se atoms have a smaller magnetic moment by $\sim 0.2-0.3 \mu_{B}$. Finally, as regards the $\operatorname{Ir}(111)$ substrate, the Fe layer induces in the Ir one underneath a small magnetic moment of $\sim 0.07 \mu_{B}$ ferromagnetically coupled to $\mathrm{Fe}$ magnetic moments while the second surface Ir layer shows an antiferromagnetic coupling.

As already discussed in the literature for molecular systems adsorbed on magnetic surfaces [53-56], in the case of the magnetic surface atoms the molecule-surface interaction can modify the magnitude of their magnetic moments as well as their interatomic distances. Importantly, these structural and electronic changes of the magnetic surface layer can also alter the strength of the magnetic exchange interactions between the surface atoms that in turn leads to a magnetic hardening or softening of the corresponding exchange coupling constants $J$ [53-55].

In consequence, for the $\mathrm{TMD} / \mathrm{Fe} / \mathrm{Ir}(111)$ systems analyzed in our theoretical study the exchange coupling constants $J_{1}$ and $J_{2}$ that describe the strength of the magnetic exchange interaction between the surface $\mathrm{Fe}$ atoms [see Fig. 1(c)] are evaluated with the help of an effective Heisenberg Hamiltonian $H=-\sum_{i>j} J_{i j} \mu_{i} \mu_{j}$ that considers only the coupling between the nearest-neighbor pairs of atoms $i$ and $j$ with the magnetic moments $\mu_{i}$ and $\mu_{j}$, respectively. The magnetic exchange couplings $J_{1}$ and $J_{2}$ evaluated for the $\mathrm{Fe}_{\text {surf }}^{\mathrm{S} / \mathrm{Se}}-\mathrm{Fe}_{\text {surf }}$ and $\mathrm{Fe}_{\text {surf }}^{\mathrm{S} / \mathrm{Se}}-\mathrm{Fe}_{\text {surf }}^{\mathrm{S} / \mathrm{Se}}$ (or $\mathrm{Fe}_{\text {surf }}$ coordinated by $\mathrm{S} / \mathrm{Se}$ in top-hollow sites) surface atoms are reported in Table I. As a general observation, in the case of the $\mathrm{VS}_{2} / \mathrm{Fe} / \mathrm{Ir}(111)$ and $\mathrm{VSe}_{2} / \mathrm{Fe} / \mathrm{Ir}(111)$ systems the exchange coupling constant $J_{2}$ describing the magnetic interactions between the $\mathrm{Fe}_{\text {surf }}$ atoms coordinated by the $\mathrm{S}_{\text {top }}$ and $\mathrm{S}_{\text {hollow }}\left(\mathrm{Se}_{\text {top }}\right.$ and $\left.\mathrm{Se}_{\text {hollow }}\right)$ atoms are significantly larger than the $J_{1}$ obtained for the $\mathrm{Fe}_{\text {surf }}^{\mathrm{S} / \mathrm{Se}}-\mathrm{Fe}_{\text {surf }}$ atomic pairs. On the other hand, for Mo and $\mathrm{W}$ disulfides on $\mathrm{Fe} / \mathrm{Ir}(111)$ the magnetic exchange interactions between the $\mathrm{Fe}_{\text {surf }}^{\mathrm{S}}$ atoms with atop $\mathrm{S}$ ones as quantified by the exchange coupling $J_{2}$ are weaker with respect to those between $\mathrm{Fe}_{\text {surf }}^{\mathrm{S}}$ and $\mathrm{Fe}_{\text {surf }}$ atoms and described by the exchange coupling $J_{1}$. However, the opposite trend is observed for the diselenidebased systems. With respect to the exchange coupling constant of the clean $\mathrm{Fe} / \mathrm{Ir}(111)$ surface $J_{\mathrm{cs}}$ of $18.0 \mathrm{meV}$ [53], the calculated $J$ values indicate a hardening of the magnetic interactions for the $\mathrm{Fe}-\mathrm{Fe}$ atomic pairs. This outcome of our study is similar to the results reported for finite $\pi$ molecular systems on $\mathrm{Fe} / \mathrm{W}(110)$ [54-56] and for extended $\pi$ ones such as graphene and $\mathrm{hBN}$ on $\mathrm{Co}(111)$ [57]. As discussed in depth for these molecular-based systems, the mechanism of the magnetic hardening of the exchange coupling constants $J$ for the $\mathrm{Fe}$ surface atoms is intrinsically related to the strong hybridization between the out-of-plane $\mathrm{Fe} d$-like atomic orbitals and the electronic states of the TMD layer, especially with the interfacial $\mathrm{S}(\mathrm{Se}) p_{z}$-like ones. Therefore our present and previous results obtained for (nonmagnetic) organic molecules and (magnetic and nonmagnetic) TMDs on magnetic surfaces clearly illustrates the generality of the magnetic hardening effect related to the increase of the magnetic exchange coupling due to chemical interactions between an adsorbate and a magnetic surface of choice.

\section{SUMMARY AND OUTLOOK}

In this first-principles study we systematically investigated the electronic and magnetic properties of several magnetic and nonmagnetic TMD monolayers such as V, Mo, and W disulphides and diselenides (i.e., $\mathrm{VS}_{2}, \mathrm{MoS}_{2}, \mathrm{WS}_{2}, \mathrm{VSe}_{2}$, $\mathrm{MoSe}_{2}$, and $\mathrm{WSe}_{2}$ ) deposited on the magnetic metal electrode $\mathrm{Fe} / \mathrm{Ir}(111)$. As a common feature, all semiconductor TMDs investigated in this study acquire a metallic character upon adsorption on the Fe/Ir(111) surface due to a rather strong TMD-substrate hybridization pointing to a chemisorption bonding mechanism. From a magnetic point of view, the TMD-Fe/Ir(111) hybridization leads to a spin-imbalanced electronic structure with features specific for each hybrid system as also reflected by the simulated spin polarization maps. Interestingly, this spin-polarized hybridization process induces also a magnetic moment in the Mo and W TM atoms of the TMD monolayers that are antiferromagnetically coupled to the $\mathrm{Fe}$ atomic layer. Besides this, our theoretical investigation suggests that the interfaces obtained for magnetic TMDs such as $\mathrm{VS}_{2}$ and $\mathrm{VSe}_{2}$ strongly chemisorbed on the $\mathrm{Fe} / \mathrm{Ir}(111)$ magnetic substrate may be better suited for spin transport than the nonmagnetic ones (i) due to a strong spin-dependent hybridization between the $\mathrm{Fe}$ and $\mathrm{V} d$ states near Fermi energy via the $p$ ones of the $\mathrm{S}$ (Se) interfacial atomic layer as well as (ii) due to the formation of an interface Ohmic contact. Furthermore, at the TMD-Fe/Ir(111) interface the strength of the magnetic interactions between the $\mathrm{Fe}$ surface atoms as described by the exchange coupling constants evaluated within the Heisenberg model are significantly increased with respect to that obtained for the clean Fe/Ir(111) surface. Interestingly, this magnetic hardening effect of the exchange coupling constants is similar to that revealed for hybrid organic-magnetic surface systems and therefore demonstrates the generality of this adsorbate-induced effect on magnetic substrates. Overall, our $a b$ initio study of hybrid interfaces obtained by wiring TMD monolayers on a metal magnetic surface is a first necessary step to investigate further properties of these system such as spin transport with the final goal to design realistic systems for 2D-based spintronic devices.

\section{ACKNOWLEDGMENTS}

The authors gratefully acknowledge the Gauss Centre for Supercomputing (GCS) for providing computing time through the John von Neumann Institute for Computing (NIC) on 
the GCS share of the supercomputer JURECA at Jülich Supercomputing Centre (JSC). This work has been supported by the Deutsche Forschungsgemeinschaft (DFG) through the Collaborative Research Center SFB 1238 (Project C01).
[1] M. Chhowalla, H. S. Shin, G. Eda, L.-J. Li, K. P. Loh, and H. Zhang, Nat. Chem. 5, 263 (2013).

[2] X. Xu, W. Yao, D. Xiao, and T. F. Heinz, Nat. Phys. 10, 343 (2014).

[3] Q. H. Wang, K. Kalantar-Zadeh, A. Kis, J. N. Coleman, and M. S. Strano, Nat. Nanotechnol. 7, 699 (2012).

[4] G. Fiori, F. Bonaccorso, G. Iannaccone, T. Palacios, D. Neumaier, A. Seabaugh, S. K. Banerjee, and L. Colombo, Nat. Nanotechnol. 9, 768 (2014).

[5] K. S. Novoselov, A. K. Geim, S. V. Morozov, D. Jiang, Y. Zhang, S. V. Dubonos, I. V. Grigorieva, and A. A. Firsov, Science 306, 666 (2004).

[6] K. S. Novoselov, V. I. Fal'ko, L. Colombo, P. R. Gellert, M. G. Schwab, and K. Kim, Nature (London) 490, 192 (2012).

[7] B. Radisavljevic, A. Radenovic, J. Brivio, V. Giacometti, and A. Kis, Nat. Nanotechnol. 6, 147 (2011).

[8] B. W. H. Baugher, H. O. H. Churchill, Y. Yang, and P. JarilloHerrero, Nat. Nanotechnol. 9, 262 (2014).

[9] J. S. Ross, P. Klement, A. M. Jones, N. J. Ghimire, J. Yan, D. G. Mandrus, T. Taniguchi, K. Watanabe, K. Kitamura, W. Yao, D. H. Cobden, and X. Xu, Nat. Nanotechnol. 9, 268 (2014).

[10] H. Wang, L. Yu, Y.-H. Lee, Y. Shi, A. Hsu, M. L. Chin, L.-J. Li, M. Dubey, J. Kong, and T. Palacios, Nano Lett. 12, 4674 (2012).

[11] K. F. Mak, C. Lee, J. Hone, J. Shan, and T. F. Heinz, Phys. Rev. Lett. 105, 136805 (2010).

[12] D. Voiry, A. Goswami, R. Kappera, C. de Carvalho Castro e Silva, D. Kaplan, T. Fujita, M. Chen, T. Asefa, and M. Chhowalla, Nat. Chem. 7, 45 (2015).

[13] D. Xiao, G.-B. Liu, W. Feng, X. Xu, and W. Yao, Phys. Rev. Lett. 108, 196802 (2012).

[14] H. Zeng, J. Dai, W. Yao, D. Xiao, and X. Cui, Nat. Nanotechnol. 7, 490 (2012).

[15] K. F. Mak, K. He, J. Shan, and T. F. Heinz, Nat. Nanotechnol. 7, 494 (2012)

[16] G. Aivazian, Z. Gong, A. M. Jones, R.-L. Chu, J. Yan, D. G. Mandrus, C. Zhang, D. Cobden, W. Yao, and X. Xu, Nat. Phys. 11, 148 (2015).

[17] G. Wang, C. Robert, A. Suslu, B. Chen, S. Yang, S. Alamdari, I. C. Gerber, T. Amand, X. Marie, S. Tongay, and B. Urbaszek, Nat. Commun. 6, 10110 (2015).

[18] X. Qian, J. Liu, L. Fu, and J. Li, Science 346, 1344 (2014).

[19] R. Kappera, D. Voiry, S. E. Yalcin, B. Branch, G. Gupta, A. D. Mohite, and M. Chhowalla, Nat. Mater. 13, 1128 (2014).

[20] A. Allain, J. Kang, K. Banerjee, and A. Kis, Nat. Mater. 14, 1195 (2015).

[21] S. Xu, Z. Wu, H. Lu, Y. Han, G. Long, X. Chen, T. Han, W. Ye, Y. Wu, J. Lin, J. Shen, Y. Cai, Y. He, F. Zhang, R. Lortz, C. Cheng, and N. Wang, 2D Mater. 3, 021007 (2016).

[22] W. Chen, E. J. G. Santos, W. Zhu, E. Kaxiras, and Z. Zhang, Nano Lett. 13, 509 (2013).

[23] C. Gong, L. Colombo, R. M. Wallace, and K. Cho, Nano Lett. 14, 1714 (2014).

[24] D. Çakir and F. M. Peeters, Phys. Rev. B 89, 245403 (2014).
[25] Y. Guo, D. Liu, and J. Robertson, ACS Appl. Mater. Interfaces 7, 25709 (2015).

[26] H. Zhong, R. Quhe, Y. Wang, Z. Ni, M. Ye, Z. Song, Y. Pan, J. Yang, L. Yang, M. Lei, J. Shi, and J. Lu, Sci. Rep. 6, 21786 (2016).

[27] J. Kang, W. Liu, D. Sarkar, D. Jena, and K. Banerjee, Phys. Rev. X 4, 031005 (2014).

[28] B. A. Helfrecht, D. M. Guzman, N. Onofrio, and A. H. Strachan, Phys. Rev. Mater. 1, 034001 (2017).

[29] I. Žutić, J. Fabian, and S. D. Sarma, Rev. Mod. Phys. 76, 323 (2004).

[30] O. L. Sanchez, D. Ovchinnikov, S. Misra, A. Allain, and A. Kis, Nano Lett. 16, 5792 (2016).

[31] W. Wang, A. Narayan, L. Tang, K. Dolui, Y. Liu, X. Yuan, Y. Jin, Y. Wu, I. Rungger, S. Sanvito, and F. Xiu, Nano Lett. 15, 5261 (2015).

[32] H.-C. Wu, C. O. Coileái, M. Abid, O. Mauit, A. Syrlybekov, A. Khalid, H. Xu, R. Gatensby, J. J. Wang, H. Liu, L. Yang, G. S. Duesberg, H.-Z. Zhang, M. Abid, and I. V. Shvets, Sci. Rep. 5, 15984 (2015).

[33] A. Kuc, N. Zibouche, and T. Heine, Phys. Rev. B 83, 245213 (2011).

[34] Z. Y. Zhu, Y. C. Cheng, and U. Schwingenschlögl, Phys. Rev. B 84, 153402 (2011).

[35] Y. Li, S. Tongay, Q. Yue, J. Kang, J. Wu, and J. Li, Appl. Phys. Lett. 114, 174307 (2013).

[36] X. Tian, L. Liu, Y. Du, J. Gu, J.-b. Xu, and B. I. Yakobson, Phys. Chem. Chem. Phys. 17, 1831 (2015).

[37] H. Pan, J. Phys. Chem. C 118, 13248 (2014).

[38] G. C. Loh and R. Pandey, Phys. Chem. Chem. Phys. 17, 18843 (2015).

[39] Y. Zhou, Q. Su, Z. Wang, H. Deng, and X. Zu, Phys. Chem. Chem. Phys 15, 18464 (2013).

[40] Y. C. Cheng, Q. Y. Zhang, and U. Schwingenschlögl, Phys. Rev. B 89, 155429 (2014).

[41] A. N. Andriotis and M. Menon, Phys. Rev. B 90, 125304 (2014).

[42] H. Shi, H. Pan, Y.-W. Zhang, and B. I. Yakobson, Phys. Rev. B 88, 205305 (2013).

[43] P. Manchanda, V. Sharma, H. Yu, D. J. Sellmyer, and R. Skomski, Appl. Phys. Lett. 107, 032402 (2015).

[44] H. Guo, N. Lu, L. Wang, X. Wu, and X. C. Zeng, J. Phys. Chem. C 118, 7242 (2014).

[45] X. Zhang, W. Mi, X. Wang, Y. Cheng, and U. Schwingenschlögl, Sci. Rep. 4, 7368 (2014).

[46] M. Y. Yin, X. C. Wang, W. B. Mi, and B. H. Yang, Comput. Mater. Sci. 99, 326 (2015).

[47] Q. Chen, Y. Ouyang, S. Yuan, R. Li, and J. Wang, ACS Appl. Mater. Interfaces 6, 16835 (2014).

[48] C. Song, S. Gong, Z. Zhang, H. Mao, Q. Zhao, J. Wang, and H. Xing, J. Phys. D: Appl. Phys. 48, 485001 (2015).

[49] K.-A. Min, J. Cha, K. Cho, and S. Hong, 2D Mater. 4, 024006 (2017).

[50] T. Garandel, R. Arras, X. Marie, P. Renucci, and L. Calmels, Phys. Rev. B 95, 075402 (2017). 
[51] J.-R. Chen, P. M. Odenthal, A. G. Swartz, G. C. Floyd, H. Wen, K. Y. Luo, and R. K. Kawakami, Nano Lett. 13, 3106 (2013).

[52] S. Heinze, K. von Bergmann, M. Menzel, J. Brede, A. Kubetzka, R. Wiesendanger, G. Bihlmayer, and S. Blügel, Nat. Phys. 7, 713 (2011).

[53] J. Brede, N. Atodiresei, V. Caciuc, M. Bazarnik, A. Al-Zubi, S. Blügel, and R. Wiesendanger, Nat. Nanotechnol. 9, 1018 (2014).

[54] M. Callsen, V. Caciuc, N. Kiselev, N. Atodiresei, and S. Blügel, Phys. Rev. Lett. 111, 106805 (2013).

[55] R. Friedrich, V. Caciuc, N. S. Kiselev, N. Atodiresei, and S. Blügel, Phys. Rev. B 91, 115432 (2015).

[56] R. Friedrich, V. Caciuc, N. Atodiresei, and S. Blügel, Phys. Rev. B 92, 195407 (2015).

[57] R. Friedrich, V. Caciuc, N. Atodiresei, and S. Blügel, Phys. Rev. B 93, 220406(R) (2016).

[58] P. Hohenberg and W. Kohn, Phys. Rev. 136, B864 (1964).

[59] W. Kohn and L. J. Sham, Phys. Rev. 140, A1133 (1965).
[60] J. P. Perdew, K. Burke, and M. Ernzerhof, Phys. Rev. Lett. 77, 3865 (1996).

[61] P. E. Blöchl, Phys. Rev. B 50, 17953 (1994).

[62] G. Kresse and J. Hafner, Phys. Rev. B 47, 558 (1993).

[63] G. Kresse and J. Furthmüller, Phys. Rev. B 54, 11169 (1996).

[64] G. Kresse and D. Joubert, Phys. Rev. B 59, 1758 (1999).

[65] See Supplemental Material at http://link.aps.org/supplemental/ 10.1103/PhysRevMaterials.2.084001 for details of the SPPDOS obtained for the $\mathrm{S}, \mathrm{Se}$, and Fe atoms of all TMD/Fe/Ir(111) systems investigated in our study. Additionally, the planeaveraged electrostatic potentials $\langle V\rangle$ obtained for planes parallel to the TMD-surface interfaces are also presented.

[66] C. Barraud, P. Seneor, R. Mattana, S. Fusil, K. Bouzehouane, C. Deranlot, P. Graziosi, L. Hueso, I. Bergenti, V. Dediu, F. Petroff, and A. Fert, Nat. Phys. 6, 615 (2010).

[67] K. V. Raman, A. M. Kamerbeek, A. Mukherjee, N. Atodiresei, T. K. Sen, P. Lazić, V. Caciuc, R. Michel, D. Stalke, S. K. Mandal, S. Blügel, M. Münzenberg, and J. S. Moodera, Nature (London) 493, 509 (2013). 